\title{
Overprescribing of Topical Ocular Corticosteroids and Antibiotics in Out-of-Hours Primary Care in Belgium
}

\author{
Hans De Loof ${ }^{\prime}$ \\ Ellen De Win' \\ Nathalie Moens' \\ Veronique Verhoeven (iD ${ }^{2}$ \\ Paul Van Royen (iD ${ }^{2}$ \\ Elke O Kreps ${ }^{3}$ \\ Hilde Philips ${ }^{2}$ \\ 'Laboratory of Physiopharmacology, \\ Department of Pharmaceutical Sciences, \\ University of Antwerp, Antwerp, \\ Belgium; ${ }^{2}$ Research Group, Primary and \\ Interdisciplinary Care (ELIZA), \\ Department Family Medicine and \\ Population Health (FAMPOP) University \\ of Antwerp, Antwerp, Belgium; \\ ${ }^{3}$ Department of Ophthalmology, Ghent \\ University Hospital, Ghent, Belgium
}

Correspondence: Hans De Loof Laboratory of Physiopharmacology, Department of Pharmaceutical Sciences, University of Antwerp, Universiteitsplein

I, Antwerp, B-26I0, Belgium

Tel +3232659l 39

Email hans.deloof@uantwerpen.be

\begin{abstract}
Prescribing patterns by primary care physicians concerning ophthalmic problems were studied using the iCAREdata, a database containing information from the out-of-hours care setting in the Flanders region of Belgium. A very high percentage of prescribed ophthalmic medication was topical antibiotics (89.4\%) with tobramycin as the most prevalent substance and in clear conflict with the prevailing guidelines. In addition, a very substantial fraction of prescribed medication contained corticosteroids $(30.4 \%)$. This is a potentially unsafe option within the technical infrastructure of this setting, which limits the diagnostic possibilities concerning viral infections or preexisting glaucoma risk. We conclude that more efforts are required to limit unnecessary and inappropriate prescribing behavior to further promote patient safety.
\end{abstract}

Keywords: antibiotics, conjunctivitis, drug utilization research, referral process

\section{Introduction}

In the context of studying the referral process in primary care of patients with eye problems, we analyzed the prescription of topical ophthalmic medication during out-of-hours care. This research was motivated by the results of a survey amongst ophthalmologists, in which they indicated that certain medications, in particular topical corticosteroids, were often inappropriately prescribed in primary care and, in the absence of formal referral guidelines, this was undermining their confidence in referrals from primary care physicians (De Win, 2019, Master thesis, Antwerp University). The use of topical antibiotics and the adherence to existing guidelines have received attention in the literature ${ }^{1,2}$ as a part of the efforts to reduce the spread of antibiotic resistance. In contrast, the frequency of use of topical corticosteroids is much less studied with the exception of the study in the US by Shekhawat et al. ${ }^{3}$ The hazards of prescribing topical steroids in the primary care setting, with its limited technical diagnostic possibilities concerning viral infections, are widely acknowledged, ${ }^{2,4}$ although not universally accepted. ${ }^{5}$ We report on the prescribing pattern of topical corticosteroid, antibiotics and their combination in out-of-hours care in Belgium.

\section{Materials and Methods}

We used the iCAREdata $^{6}$ resource for this retrospective study. This database contains the reason of encounter and the prescribed medication compiled from a 
number of out-of-hours centers in the Flemish part of Belgium. Primary care physicians take turns to work in these centers on weekends and out-of-office hours. We analyzed the iCAREdata from 2018 to 2019 containing 181,631 diagnoses. We decided not to use the 2020 data because of the COVID-19 pandemic as the COVID-19 pandemic dramatically impacted the organization of outof-hours care. ${ }^{7}$ The number of out-of-hours centers connected to iCAREdata increased between 2018 and 2019 and these years were also analyzed separately. Eye problems coded with ICPC code $\mathrm{F}$ and prescribed medicines with WHO-ATC codes S01 and S03 were extracted from the database. Products without an ATC code such as borderline medical devices, including nearly all the artificial tears, are not included in the iCAREdata database.

Modalities for the use of iCAREdata were described by Colliers et $\mathrm{al}^{6}$ and received approvals concerning patients participation and opt-out options from the Sectoral Committee of Social Security and Health of the Privacy
Commission (Beraadslaging_AG_094_2014 and Beraadslaging_AG_094_2014bis) and from the local IRB (Ethics Committee of the Antwerp Academic Hospital Approval 13/34/330, dd 02/09/2013) allowing the use of aggregated anonymized data without the need for a new approval for this particular study.

\section{Results}

Table 1 shows the results of the analysis. In this two-year period, a total of 4529 diagnoses recorded in the database were eye-related problems, corresponding to $2.49 \%$ of the caseload. The diagnoses occurring within this group, in any year, beyond $10 \%$ and the incidences of allergic conjunctivitis (ICPC code F71) are detailed in Table 1. The most common diagnoses are clearly infection related, and allergic conjunctivitis, at an average of less than $2 \%$, is not among the top 10 diagnoses. Of these contacts, $77.2 \%$ resulted in a prescription of medication and in total 5303 products were prescribed. Of these, 3695 (69.7\%) had an

Table I Prescribing Frequency of Medicines Among People Diagnosed with an Eye Problem in Out of Hours Car in Belgium

\begin{tabular}{|c|c|c|c|c|c|c|}
\hline & \multicolumn{2}{|c|}{2018} & \multicolumn{2}{|c|}{2019} & \multicolumn{2}{|c|}{ Total } \\
\hline & $\mathbf{n}$ & $\%$ & $\mathbf{n}$ & $\%$ & $\mathbf{n}$ & $\%$ \\
\hline Patient contacts & 67,447 & & 114,184 & & $|81,63|$ & \\
\hline Patient contacts with eye problems & 1542 & $2.28 \%$ & 2987 & $2.62 \%$ & 4529 & $2.49 \%$ \\
\hline \multicolumn{7}{|l|}{ Selected Diagnoses (ICPC Code) } \\
\hline Conjunctivitis infectious (F70) & 592 & $38.39 \%$ & $|28|$ & $42.89 \%$ & 1873 & $41.36 \%$ \\
\hline Conjunctivitis allergic (F7I) & 24 & $1.56 \%$ & 38 & $1.27 \%$ & 62 & $1.37 \%$ \\
\hline Blepharitis/stye/chalazion (F72) & 176 & $11.41 \%$ & 360 & $12.05 \%$ & 536 & $11.83 \%$ \\
\hline Injury eye other (F79) & 80 & $5.19 \%$ & 327 & $10.95 \%$ & 407 & $8.99 \%$ \\
\hline Eye/adnexa disease other (F99) & 300 & $19.46 \%$ & 264 & $8.84 \%$ & 564 & $12.45 \%$ \\
\hline Ophthalmic medications prescribed & $|28|$ & $81.13 \%$ & 2414 & $64.82 \%$ & 3695 & $69.68 \%$ \\
\hline Contacts resulting in a prescription & 1201 & $77.89 \%$ & 2295 & $76.83 \%$ & 3496 & $77.19 \%$ \\
\hline \multicolumn{7}{|l|}{ Prescription for } \\
\hline NSAID & 64 & $5.00 \%$ & 117 & $4.85 \%$ & 181 & $4.90 \%$ \\
\hline Antihistamines + cromoglycate & 28 & $2.19 \%$ & 53 & $2.20 \%$ & 81 & $2.19 \%$ \\
\hline Antibiotics without corticosteroids & 1146 & $89.46 \%$ & 2159 & $89.44 \%$ & 3305 & $89.45 \%$ \\
\hline Tobramycin & 276 & $21.55 \%$ & 590 & $24.44 \%$ & 866 & $23.44 \%$ \\
\hline Fusidic acid & 253 & $19.75 \%$ & 455 & $18.85 \%$ & 708 & $19.16 \%$ \\
\hline Tetracycline containing products & 109 & $8.51 \%$ & 209 & $8.66 \%$ & 318 & $8.61 \%$ \\
\hline Quinolones & 64 & $5.00 \%$ & 129 & $5.34 \%$ & 193 & $5.22 \%$ \\
\hline Corticoids. all combinations with $A B$ & 407 & $31.77 \%$ & 718 & $29.74 \%$ & 1125 & $30.45 \%$ \\
\hline Corticoids. excluding hydrocortisone & 345 & $26.93 \%$ & 592 & $24.52 \%$ & 937 & $25.36 \%$ \\
\hline Corticoids without antibiotics & 9 & $0.70 \%$ & 19 & $0.79 \%$ & 28 & $0.76 \%$ \\
\hline
\end{tabular}


ophthalmologic WHO-ATC code corresponding to a topical ophthalmic medication.

A very high proportion (89.4\%) of these prescriptions for topical eye products contained antibiotics and $62.3 \%$ of all diagnosed eye problems resulted in the prescribing of an antibiotic. Comparing the products containing only an antibiotic, nearly one in four of all prescriptions was for tobramycin $(23.4 \%)$, exceeding the frequency of fusidic acid $(19.2 \%)$, tetracycline containing products $(8.6 \%)$ and quinolones $(5.2 \%)$.

In addition, we observed a high proportion of products containing corticosteroids amounting $30.4 \%$ of all prescriptions. More than one in five diagnosed eye problems resulted in a corticosteroid prescription $(21.2 \%)$. Of these, 1125 corticoid prescriptions, $97.5 \%$ were for combination products with antibiotics. Of these combinations, 188 contained hydrocortisone, and all other combination products contained stronger compounds, mainly dexamethasone.

\section{Discussion}

Prescribing patterns by primary care physicians, responding to eye problems in the out-of-hours care in Flanders (Belgium), were very similar across the two consecutive yearly periods. The bigger sample in 2019 did not result in noticeable differences in the frequencies of prescribed products. Prescribing primary care physicians represented a large and representative sample of the current workforce. $^{7}$

Chloramphenicol is very rarely prescribed, likely due to persistent unavailability issues. The prescription frequency of artificial tears was very low in iCAREdata as these first-line products are mostly medical devices and thus not captured in the database limited to medicines with an ATC code. We therefore cannot comment on their usage. Another aspect to bear in mind is that we studied prescription data, and we cannot yet couple this with dispensing data.

It is quite obvious that the observed prescription pattern is difficult to match with the Belgian BAPCOC guidelines on the use of antibiotics in primary care. ${ }^{8}$ According to these guidelines, a tetracycline or fusidic acid-based preparation are the preferred products ${ }^{8}$ if an antibiotic is needed for the treatment of bacterial conjunctivitis. These two compounds together represent less than half of the antibiotic-only products, whereas tobramycin and quinolones encompass the majority of these prescriptions. One in five patients diagnosed with an eye problem receives a prescription for one of these second-line antibiotics. We note that high-frequency antibiotics prescribing has been observed previously ${ }^{1}$ in other settings. One mitigating factor could be the over-the-counter (OTC) availability of the two recommended products. Primary care physicians may be biased against their use under the assumption that they have already been applied. Better data-exchange within primary care between physicians and pharmacists could mitigate this problem. We note that in 2021, after the study period, the BAPCOC guidelines were updated. There were, however, no changes that might have been anticipated by the physicians and could have justified the observed antibiotic prescription pattern. Guidelines should not be rigid mandates, and we acknowledge the existence of a limited number of cases with a good rationale for deviating from them. This, however, cannot justify the observed big departure from the guidelines.

The most noticeable result, however, is the high-frequency prescribing of corticoid-containing products, of which most contain a strong compound such as dexamethasone. One in five patients presenting at the out-of-hours setting received a prescription for corticoid containing eye products. Nearly one in three medications prescribed thus contains a corticoid, mostly in combination with an antibiotic. The only indication of topical steroid use in primary out-of-hours care is severe allergic conjunctivitis, which represents a small proportion of acute conjunctivitis in primary care, ${ }^{2}$ and allergic conjunctivitis was not among the top-10 diagnoses in this population. In all other cases, such as infectious conjunctivitis, the use of topical corticosteroids is contra-indicated, which renders our findings even more worrisome. The absence of the technical infrastructure to confidently exclude herpes infections and preexistent elevated intraocular pressure or glaucomatous optic disc changes clearly jeopardizes the evaluations of the risk/benefit ratio before prescribing these products. Topical corticosteroids, particularly dexamethasone formulations, have a known risk of elevated intraocular pressure and risk of glaucoma. ${ }^{9}$ This risk is mainly associated with prolonged, frequent use of highly potent steroids, such as dexamethasone, but may occur even in short-term use ${ }^{9}$. One might expect that in an out-of-hours setting, the duration of topical corticosteroid use may be relatively short, but we lack the required data to document this. These worrisome data thus seem to confirm the opinions of ophthalmologists and undermine the confidence of the referral process in primary care. However, it may also reflect the existence of a barrier, during out-of-hours care, for easy and expeditious referral to ophthalmologists. 


\section{Conclusion}

Our data illustrate that the existence per se of guidelines does not suffice to avoid antibiotic and corticosteroid overuse in primary care. As proposed by others, ${ }^{3}$ more effort is required in educating patients, health care providers and policy makers in order to limit unnecessary and inappropriate prescribing behavior. These data corroborate the opinions expressed by ophthalmologists and these policy actions therefore could also strengthen the confidence in the overall referral process in primary care.

\section{Abbreviations}

AB, Antibiotic; ATC, Anatomical Therapeutic Chemical Classification System; ICPC, International Classification of Primary Care; NSAID, Non-Steroidal AntiInflammatory Drug.

\section{Disclosure}

The authors report grants from the Research Foundation Flanders, during the conduct of the study. The authors report no other conflicts of interest in this work.

\section{References}

1. Visscher KL, Hutnik CM, Thomas M. Evidence-based treatment of acute infective conjunctivitis: breaking the cycle of antibiotic prescribing. Can Fam Physician. 2009;55(11):1071-1075.

2. Varu DM, Rhee MK, Akpek EK, et al. Conjunctivitis preferred practice pattern ${ }^{\circledR}$. Ophthalmology. 2019;126(1):P94-P169. doi:10.1016/j. ophtha.2018.10.020

3. Shekhawat NS, Shtein RM, Blachley TS, Stein JD. Antibiotic prescription fills for acute conjunctivitis among enrollees in a large United States managed care network. Ophthalmology. 2017;124(8):10991107. doi:10.1016/j.ophtha.2017.04.034

4. Vafidis GB, Anne L, Barnes B. Steroids and the eye. Available from: https://www.rnib.org.uk/sites/default/files/GP\%20factsheet $\%$ 20-\%20Steroids\%20and\%20the\%20eye.pdf. Accessed August 4, 2021.

5. Holland EJ, Fingeret M, Mah FS. Use of topical steroids in conjunctivitis: a review of the evidence. Cornea. 2019;38(8):1062-1067. doi:10.1097/ICO.0000000000001982

6. Colliers A, Bartholomeeusen S, Remmen R, et al. Improving Care And Research Electronic Data Trust Antwerp (iCAREdata): a research database of linked data on out-of-hours primary care. BMC Res Notes. 2016;9(1):259. doi:10.1186/s13104-016-2055-x

7. Morreel S, Philips H, Verhoeven V, Tu W-J. Organisation and characteristics of out-of-hours primary care during a COVID-19 outbreak: a real-time observational study. PLoS One. 2020;15(8):e237629. doi:10.1371/journal.pone. 0237629

8. BAPCOC. .Belgian guide to anti-infective treatment in outpatient practice. Available from: https://www.bcfi.be/legacy_assets/antibiotica gids-nl.pdf. Accessed August 4, 2021.

9. Roberti G, Oddone F, Agnifili L, et al. Steroid-induced glaucoma: Epidemiology, pathophysiology, and clinical management. Surv Ophthalmol. 2020;65(4):458-472. doi:10.1016/j.survophthal.2020.01.002
Drug, Healthcare and Patient Safety

\section{Publish your work in this journal}

Drug, Healthcare and Patient Safety is an international, peer-reviewed open-access journal exploring patient safety issues in the healthcare continuum from diagnostic and screening interventions through to treatment, drug therapy and surgery. The journal is characterized by the rapid reporting of reviews, original research, clinical, epidemiological and post-marketing surveillance studies, risk management, health literacy and educational programs across all areas of healthcare delivery. The manuscript management system is completely online and includes a very quick and fair peer-review system. Visit http://www.dovepress.com/testimonials.php to read real quotes from published authors. 